\title{
Towards a Direct Visualization of Charge Transfer in Monolayer Hexagonal Boron Nitride using a Fast Pixelated Detector in the Scanning Transmission Electron Microscope
}

\author{
G.T. Martinez ${ }^{1}$, T. J. Pennycook ${ }^{2}$, T.C. Naginey ${ }^{1}$, L. Jones ${ }^{1}$, H. Yang ${ }^{3}$, J.R. Yates ${ }^{1}$, R.J. Nicholls ${ }^{1}$, M. \\ Huth $^{2}$, M. Simson ${ }^{2}$, H. Soltau ${ }^{3}$, L. Strüder ${ }^{3}$, Y. Kondo ${ }^{4}$, R. Sagawa ${ }^{4}$, P.D. Nellist ${ }^{1}$ \\ 1. Department of Materials, Oxford University, United Kingdom \\ 2. Faculty of Physics, University of Vienna, Austria \\ ${ }^{3 .}$ Lawrence Berkeley National Laboratory, USA \\ 4. PNDetector GmbH, Germany \\ 5. PNSensor GmbH, Germany \\ 6. JEOL Ltd., Japan
}

Recent developments of fast pixelated detectors, such as the pnCCD (S)TEM camera developed by PNDetector, have enabled the acquisition of 4D-datasets that contain the full convergent beam electron diffraction pattern for each probe position in a scanning transmission electron microscope (STEM) experiment [1]. These type of datasets enable electron ptychography to be performed, which has been shown to be a powerful tool for phase imaging of a wide range of materials $[2,3]$. Moreover, when using methods such as the Wigner Distribution Deconvolution (WDD) algorithm [4], aberration free phase imaging of light element materials can be achieved [3]. Electron phase imaging techniques, such as high resolution transmission electron microscopy (HRTEM), can provide information on the atomic potential and the electronic charge density distribution [5], opening the possibility to acquire information about chemical bonds and ionization of atoms. However, these measurements can be affected by residual lens aberrations, which affect the image contrast and can overwhelm small signals due to charge transfer. In this work, we profit from aberration free phase imaging using WDD electron ptychography to explore the direct visualization of charge transfer in monolayer hexagonal boron nitride (hBN).

When phase imaging $\mathrm{hBN}$, it is expected that the contrast of $\mathrm{N}$ sites will be reduced due to the screening of the nuclear potential caused by the accumulation of charge transferred from the B site [5,6]. Therefore, the contrast of $\mathrm{N}$ and $\mathrm{B}$ atoms is predicted be very similar. For this study, we acquired a ptychographic dataset of monolayer $\mathrm{hBN}$ using a double corrected JEOL ARM200CF instrument fitted with a pnCCD (S)TEM camera. The acceleration voltage was $60 \mathrm{kV}$ to avoid damage and the 4D datasets were acquired at 1000 frames per second. Aberration free multislice image simulations considering the so-called independent atom model (IAM) with the experimental dose settings were calculated using the MULTEM software [7]. Figure 1 a) shows the aberration corrected experimental phase difference obtained from a WDD ptychographic reconstruction. Figure 1 b) shows the reconstruction of the simulation assuming a source size broadening effect of FWHM 1.05 Angstrom. The source size broadening was estimated to match the maximum experimental phase difference. 2D Gaussian functions were fitted to each $\mathrm{N}$ and $\mathrm{B}$ atom positions in the reconstructed phase images for both experiment and simulations. The heights of the fitted 2D Gaussian functions were used as measure of the phase difference caused by the atoms. They are plotted as histograms in Figure $1 \mathrm{c}$ ), and d), to which a Gaussian function was fitted to determine the mean difference between the phase difference distributions. From histogram $1 \mathrm{c}$ ), the mean difference between phase differences at $\mathrm{N}$ and $\mathrm{B}$ sites is only $2.3 \mathrm{mrad}$. For the case of simulations, a mean difference of $5.5 \mathrm{mrad}$ phase difference is obtained and the distributions are separated enough that the phase difference caused either by $\mathrm{N}$ or $\mathrm{B}$ can be 
identified. Therefore, the experimental measurements indicate a direct visualization of charge transfer, since the mean difference between $\mathrm{N}$ and $\mathrm{B}$ sites is smaller than the one expected from IAM simulations, which do not consider the bonding effects between atoms. Further study using DFT calculations in the simulations to consider charge transfer for confirmation is work in progress [8].

References:

[1] H. Ryll et al, Journal of Instrumentation 11 (2016) p. P04006

[2] P.D. Nellist, B.C. McCallum and J.M. Rodenburg, Nature 374 (1995) p. 630

[3] H. Yang et al, Nature Communications 7 (2016) p. 12532

[4] J. M. Rodenburg and R. H. Bates, Phil. Trans. R. Soc. Lond. A. 339 (1992) p. 521

[5] J.C. Meyer et al, Nature Materials 10 (2011) p. 209

[6] L. Pauling, Proc. Natl. Acad. Sci. USA 56 (1966) p. 1646

[7] I. Lobato and D. Van Dyck, Ultramicroscopy 156 (2015) p. 9

[8] The research leading to these results has received funding from the EPSRC (EP/M010708/1).

a)

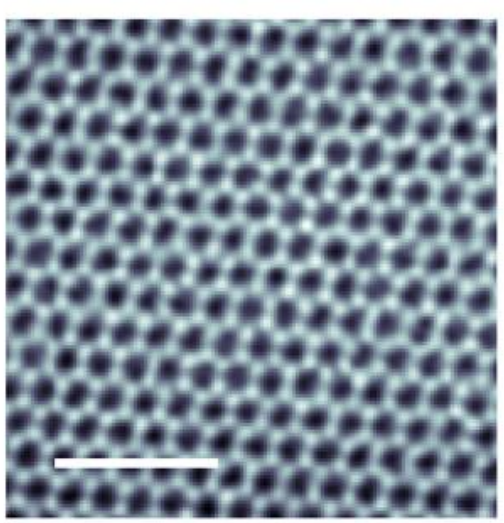

b)

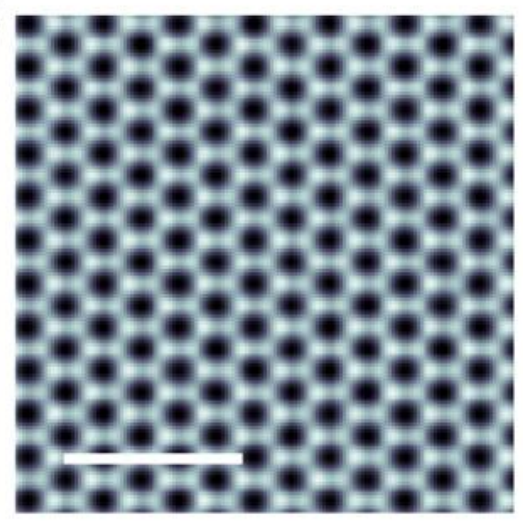

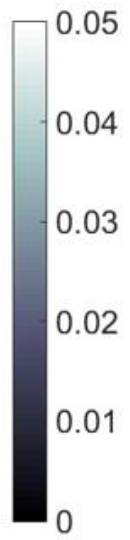

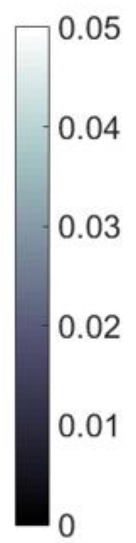

c)

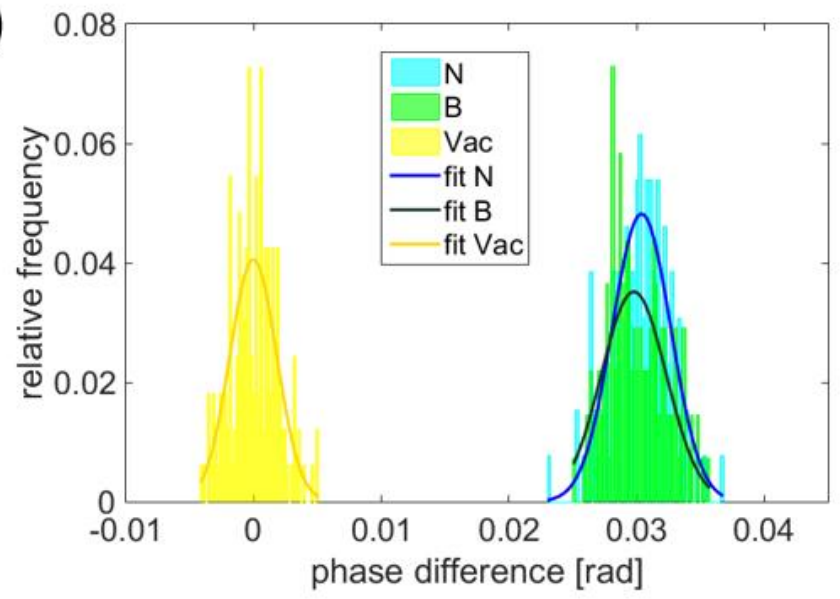

d)

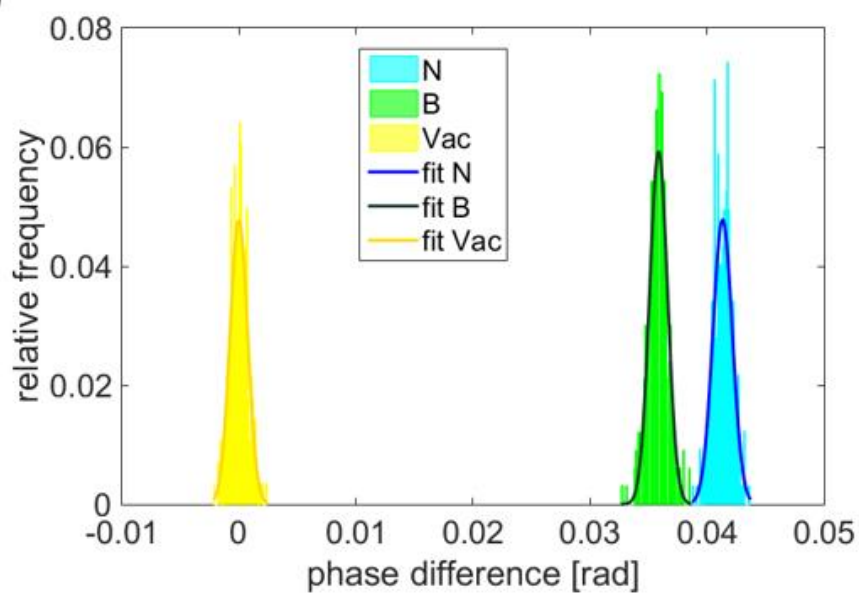

Figure 1: Aberration free phase image of monolayer hexagonal boron nitride using WDD electron ptychography a) experimental, b) simulated. Simulation assumed a source size broadening FWHM of 1.05 Angstrom. Scale bar corresponds to $1 \mathrm{~nm}$. Histograms of phase differences for $\mathrm{N}$ and B sites and vacuum region as reference with overlay of corresponding Gaussian fits for c) experimental and d) simulation. 